\title{
Kasuistischer Beitrag zur operativen Behandlung der Trigeminusneuralgie nach Krause $\mathrm{P}^{1}$ ).
}

\author{
Von Dr. Eugen Bircher in Aarau.
}

(Mit 4 Abbildungen.)

Die intrakranielle Exstirpation des Ganglion Gasseri ist noch nicht eine so alltägliche Operation geworden, als daß es sich nicht verlohnen dürfte, auch hier noch kurze kasuistische Beiträge niederzulegen, bei uns in der Schweiz scheint die Operation nicht besonders großen Anklang gefunden zu haben, - was bei der Schwere des Angriffs wohl zu begreifen ist - . und es sind, so viel wir erfahren konnten, erst ca. ein Dutzend Fälle operiert worden, so daß auch unsere geübtesten Operateure nicht in der Lage sind, über ähnliche Reihen von Operationen zu verfügen, wie $\mathrm{z} . \mathrm{B}$. K ra use mit 64 , Doll inge r mit 22 Fällen.

Immerhin wird der neurologisch tätige Arzt hie und da in die Lage kommen, sein Urteil über den Eingriff abgeben zu müssen, und es erscheint uns daher nicht überflüssig, drei Fälle zu besprechen, die wir kurz hintereinander zu operieren Gelegenheit hatten, und die alle günstigen und ungünstigen Folgen des Eingriffes deutlich illustrieren, und auf diese Weise eine objektive Beurteilung des Krauseschen Eingriffes gestatten.

Jedem Arzte, der Fälle von beginnender oder ausgesprochener Trigeminusneuralgie zu behandeln Gelegenheit gehabt hat, weiß, wie hartnäckig die Affektion der medikamentären Behandlung trotzt, und die große Menge der zur Heilung angegebenen Mittel beweist zur Genüge, wie unsicher sie in ihrem Enderfolge sind. Auch die neuerdings so warm empfohlenen Alkoholinjektionen nach Schlösser, oder indifferenter Flüssigkeiten nach

I) Nach einer klinischen Demonstration der Schweiz. neurolog. Gesellschaft zu Basel, I2. Nov. I9IO. 
L a n g e, scheint auch nicht das zu leisten, was nach dem ersten Bekanntwerden der günstigen Frfolge davon erwartet wurde.

So fällt denn eine ganze große Zahl der an Trigeminusneuralgie Erkrankten der Chirurgie zur Behandlung anheim. Aber auch die Chirurgie war lange Zeit nicht in der Lage, hier glänzende Erfolge aufzuweisen. Es würde zu weit führen, alle die chirurgischen Maßnahmen zu besprechen, die im Laufe der Zeit zur Bekämpfung der Trigeminusneuralgie angewandt wurden. Es sei nur erwähnt, dab die von Albin empfohlene, von Schlichting I748 zum erstenmale ausgeführte Neurotomie des Trigeminus, ebenso diejenige des Facialis (K 1 e i $n$ ), die Nervendehnung von K l e i $n$ und $\mathrm{NuBb}$ a u $\mathrm{m}$ völlig absolet geworden sind. Auch die eine Zeitlang in Aufschwung gekommene Carotisligatur wird kaum mehr ausgeführt.

In Anwendung befinden sich heutzutage nur noch drei Verfahren: I. Die Nervenextraktion nach Thiersch, bei der die peripheren Trigeminusäste (supra- und infraorbitalis und mandibularis) freigelegt und ausgerissen werden. Während Thiersch selber mit dieser Methode recht ordentliche Resultate erzielte, indem 30 Proz. Dauerheilungen und 30 Proz. wesentliche Besserungen nachgewiesen werden konnten, so erzielten andere Autoren wenige günstige Resultate. Busch konnte aus der v. B erg m a n n schen Klinik nur über 29,2 Proz. Heilungen berichten, Kra use sah 70 Proz. Rezidive. Über ganz schlechte Resultate berichtet $\mathrm{Hull}$ es aus der v. Eiselsbergschen Klinik, der bei 2 I Fällen gar keine Dauerresultate konstatieren konnte, und durchschnittlich nach 6-r2 Monaten Rezidive auftreten sah. Etwas bessere Resultate erhielt A n g e $\mathrm{r}$ e rs. Im allgemeinen scheint jedoch das Urteil $\mathrm{T}$ r e $\mathrm{n}$ d e le nb u rgs zuzutreffen, wonach mit der Thierschen Operation in ca. ein Drittel der Fälle Heilung oder Besserung zu erzielen ist. (Diese Zahl dürfte das Maximum darstellen.)

Die Rezidive müssen vor allem auf die große Regenerationsfähigkeit der Nerven zurückgeführt werden, so daß man häufig bei einem zweiten und dritten Eingriff nach kürzester Frist einen völlig nachgewachsenen Nervenast antreffen kann. Andrerseits dürfte die von $\mathrm{Th}$ iersch und neuerdings von $\mathrm{H}$ ulles ausgesprochene Ansicht in zahlreichen Fällen Gültigkeit haben, daß 
der Sitz der Affektion weniger in den peripheren Ästen zu suchen ist, als mehr zentralwärts im Ganglion Gasseri oder in einer abnormen Erregbarkeit sensorischer Zentren im Gehirne selbst, welche mit der Eigentümlichkeit verbunden ist, empfangene Reize anzusammeln und die angesammelte Energie in einer plötzlichen Entladung wieder abzugeben, ähnlich wie man sich bei der Epilepsie die Vorgänge an motorischen Zentren denken kann.

Auch rein anatomische zentrale Veränderungen können als Ursache der Neuralgie angesprochen werden. So fand L exer einen Tumor der hintern Schädelgrube, der den Trigeminusstamm umwachsen hatte, nach $\mathrm{Krause}$ fand $\mathrm{Schuh}$ ein Cholesteatom, das den Trigeminus umfaßte. $\mathrm{H}$ a gelsta $\mathrm{m}$ sah ein Endotheliom der mittleren Schädelgrube, Güsberg einen krebsartigen Tumor, und $\mathrm{M}$ a r $\mathrm{ch}$ a n d ein pigmentiertes Alveolarsarkom, die ganz gut als Ursache der Neuralgie angesprochen werden können. Ähnliche Beobachtungen stammen von $\mathrm{Hausch}$, $S m i t h$. Dercum und Keen, Petrinar und Klebs. Es können jetzt schon nach der Zusammenstellung von Trendelenburg eine ganze Anzahl pathologisch-anatomische Befunde, im Ganglion selbst oder dessen Umgebung nachgewiesen werden, die als Ursache der Neuralgie angesprochen werden müssen.

Daß die Neuraixerese in diesen Fällen überflüssig und nutzlos ist, ist ohne weiteres klar, aber auch die eingreifendere Resektion der Trigeminusäste II und III an der Schädeibasis, wie sie von Lüke, Camochan, v. Bruns, Pancoast, vor allem von $\mathrm{K}$ rönlein ausgeführt worden ist, kann die gewünschte Hilfe nicht bringen.

Bei diesem sehr diffizilen und an die Technik hohe Anforderungen stellenden Verfahren sieht man relativ häufig schwere Rezidive auftreten. Leider läßt uns gerade hier die Literatur im Stich, wir entnehmen einer kleineren, jedoch einwandsfreien Statistik von $\mathrm{H}$ ulles über $\mathrm{I} 3$ in der $\mathrm{E}$ is e ls berg schen Ǩlinik operierte Fälle, daß nur 3 Fälle geheilt blieben, während 5 schwere Rezidive und 5 mal kurz nach der Operation schon wieder Schmerzen auftraten. Die Rezidive folgten zwischen 6 und 24 Monaten. Unter den von $\mathrm{T}$ ü r ck 201 Fällen von Ganglionexstirpation war vorher schon $I 7$ mal an der Schädelbasis operiert worden. 
An der Schädelbasis ist es manchmal gar nicht immer möglich, sicher den 2. und 3. Ast zu resezieren. Die Blutung wird manchmal eine so erhebliche, daß man die Operation frühzeitig beenden muß. Ras u mowsky weist neuerdings darauf hin, daß die extrakranielle Resektion keineswegs leicht und ungefährlicher sei, indem der erfahrene $\mathrm{K}$ r a u s e 8,7 Proz. Mortalität hatte. Nach $R$ as u mowsky gelingt es extrakraniell nicht immer, bei der Tiefe der Operationswunde und der häufig sehr starken venösen Blutung sämtliche sensible Stämme von Ast 2 und $3 \mathrm{zu}$ fassen; er selbst erlebte einen solchen Fall, bei dem die Operation unterbrochen werden mußte. Das therapeutische Resultat ist dann kein besonders günstiges.

Als einzig in seinem Erfolge sicheres Heilmittel in der optrativen Behandlung der Trigeminusneuralgie bleibt die Entfernung des Ganglion Gasseri, wie sie I884 von Ewing Mears vorgeschlagen, von Willi a m R os e I8go zum ersten Male ausgeführt, von $\mathrm{H}$ artle $\mathrm{y}-\mathrm{Krause} \mathrm{zu}$ einem methodischen Eingriff erhoben worden ist, das heute das am meisten angewandte Verfahren ist, und die sichersten Resultate gibt: Bis zum Jahre I902 konnte $\mathrm{T} \ddot{\mathrm{u}} \mathrm{rck}$ 20I Fälle von Ganglionexstirpationen zusammenstellen, und so annähernde ein Bild von den Operationserfolgen geben, wenn auch konstatiert werden muB, daß meist nur günstig verlaufende Fälle bekanntgegeben worden sind, und diejenigen Fälle, welche neben dem günstigen Erfolge anderweitjge schwere Schädigungen erlitten, nicht immer bekannt werden.

Türck berechnet eine Mortalität von I7 Proz., während 93,6 Proz. der Úberlebenden dauernd geheilt blieben. Echte Rezidive konnte Türck nur in 4 Fällen nachweisen, während Prat 5,6 Proz., $\mathrm{Cushing} 5$ Proz. fand. Eine kurze Übersicht aus der neuern Literatur seit $\mathrm{T}$ ü r ck ergab, daß die $\mathrm{Krause}$ sche Operation seither noch $39 \mathrm{mal}$ zur Ausführung gekommen ist ( $\mathrm{P}$ o p pert, van Hook, von Eiselsberg, Helferich, Schloffer, Abbè, Schwarz, Körte u. a.). Dabei ergab sich eine Mortalität von I5 Proz., während die übrigen 33 Fälle alle geheilt worden sind. K r a u s e selbst, der die größte Erfahrung in diesen Fragen besitzt, hatte auf 5I Operationen 7 Todesfälle, nicht ganz I4 Proz. Rezidive konnte er selbst keine 
beobachten, und verfügt er doch über Fälle, bei denen über Io Jahre seit der Operation verflossen.

Die beschriebenen Fälle sollen unser Vorgehen bei der Operation erläutern, an die wir einige Erörterungen knüpfen möchten.

I. 76 jähr. Patientin, geb. I833, erster Eintritt 3. XII. I894, stammt aus sonst gesunder Familie, war selber nie krank. Die jetzige Erkrankung begann vor 7 Jahren ( 1887 ) mit anfallsweise auftretenden brennenden und stechenden Schmerzen, die in der rechten Infraorbitalgegend begannen. Die geringste Bewegung, besonders das Essen, löste heftige Schmerzanfälle aus. Die Schmerzen verbreiteten sich in die Stirn und die linke Gesichtshälfte. Besonders während der Nacht waren die Schmerzen äußerst intensiv, während in der wärmeren Jahreszeit die Schmerzanfälle zurückgingen.

Ausreißen aller Zähne und medikamentöse Behandlung blieben ohne Erfolge.

Stat us: Magere Frau, mit schmerzhaft verzogenem Gesicht. Doppelseitige Konjunctivitis. Druck auf den Infraorbitalis löst einen heftigen Schmerzanfall aus: supraorbital weniger.

D i a g n o se: Neuralgia nerv. hegemi dextra.

The rapie: Neuraixerese des Nerv. infra- und supraorbitalis. Die Schmerzen hören rechterseits prompt auf, deutliche Anästhesie, setzen dagegen nach einigen Tagen um so heftiger links ein, so da $\beta$ am I2. XII. linkerseits die Nervendehnung des Infra- und supraorbitalis ausgeführt wird, worauf die Anfälle bis zum I5. XII. I896 sistieren, wo sie von neuem äußerst heftig in der rechten Gesichtshälfte auftreten, besonders intensiv im $\mathrm{N}$. infraorbitalis, der aus dem Foramen infraorbitale ausgemeißelt und der Nerv in rollständigem Zusammenhang auf $5 \mathrm{~cm}$ Iänge ausgerissen werden kann, so daß Patientin am I4. III. geheilt entlassen wurde.

Im Januar I897 treten im Infraorbitalis links von neuem äußerst heftige Schmerzen auf, worauf am I6. 1. I897 der Nerv, der früher nur gedehnt worden war, auf eine größere Strecke in toto ausgerissen wird.

Patientin konnte nach 8 Tagen schmerzfrei entlassen werden.

Seit dem Nov. Igoo treten wiederum neue Schmerzattacken im linken Supraorbitalis, Supramaxillaris und mandibularis auf, so daß bloß durch Morphium ein erträglicher Zustand geschaffen werden kann.

Am II. XII. werden die Nerv. mandibularis und Supraorbitalis excidiert, worauf die Schmerzanfälle nachlassen und Anästhesie in den betreffenden Gebieten einsetzt.

Nach $1 / 4$ Jahr treten die Schmerzen im ersten und zweiten Ast neuerdings auf, so daß das Dach der Orbita aufgemeißelt und der Nerv tief innen ausgedreht wird.

Nun war bis zum Mai I904 ein erträglicher Zustand geschaffen, als die Schmerzen am N. supra- und infraorbitalis von neuem ein- 
setzten. Es ist eine Hyperästhesie in den Partien dieses Nerven vorhanden und die Operation zeigt auch, daß beide Nerven nachgewachsen sind und von neuem entfernt werden müssen. Patientin kann wiederum völlig schmerzfrei entlassen werden. Jetzt ist ein deutliches Ektropium und ein starktränendes Auge vorhanden.

Im Jahre I907 war Patientin einer Humerusfraktur wegen in der Anstalt. Sie hatte niemals Anfälle, die ganze linke Gesichtshälfte war anästhetisch und zeigte eine beginnende Atrophie. Bis zum Winter I909-IgIo ging es der Patientin recht ordentlich, bis im Dezember Igog die Schmerzen mit bis dahin nie dagewesener Heftigkeit einsetzten, auf kein Mittel, selbst auf große Dosen Morphium nicht sistierten, so daß Patientin lebensüberdrüssig geworden ist und auch schon einen Suizidversuch gemacht hat, und nun verlangt, in ihrem 77. Altersjahre endgültig ron ihren Schmerzen befreit zu werden und lieber sterben wolle, als auf diese Weise ihr Dasein fristen.

Die Schmerzen sind nun konstant in der ganzen linken Gesichtsund Kopfhälfte vorhanden und sistieren auf kaum 5 Min.

St a tus: Alte kräftige Frau. Herz und Lungen ohne Befund. Die Gegend des Supra- und Infraorbitalis zeigt eine deutliche Knochenatrophie und ist auf Druck kolossal schmerzempfindlich, ebenso der Ramus mandibularis. Die Sensibilität ist in diesem ganzen Gebiete vorhanden. Der Masseterenreflex fehlt. Der Gaumen ist gerötet, etwas hyperästhetisch, der Alveolarrand kolossal druckempfindlich.

Das linke Auge tränt stark, die Conjunctiva ist gerötet. Deutliche Keratitis. Patientin will schon lange auf diesem Auge schlecht gesehen haben. Hochgradiges Ektropium ist vorhanden. Der Schädel ist auf Klopfen und Druck ziemlich stark empfindlich und es können dadurch die Schmerzanfälle ausgelöst werden.

Am 30. III. I9Io Operation nach $\mathrm{K} \mathrm{r}$ a u s e in halb sitzender Lage (Operateur: Sek.-Arzt Dr. Eu gen Bircher). I. Prophylaktische Unterbindung der Carotis externa an Vorderrande des Sternocleido mastoideus. 2. Bildung eines Krauseschen Hautperiostlappens, der Jochbogen wird temporär reseziert; mit der Fräse und die Temporalgegend eröffnet und der Knochen bis an die Umschlagsstelle in die Basis in Fünffrankstückgröße entfernt. Die Dura läßt sich leicht vom Knochen abschieben und das atrophische Gehirn mit einem gynäkologischen Spatel in die Höhe heben. Die Meningea media werden durchtrennt und rasch und leicht gelangt man an Ramus III, dann II, zuletzt an das Ganglion, daß sich leicht frei präparieren läßt und mit Kornzange samt Ramus I ausgerissen wird. Die mäßige, hauptsächlich venöse Blutung wird durch Adrenalintampons zur Stillung gebracht. Die Hautwunde wird exakt vernäht und eine feine Mèche eingeführt.

Am I. IV. wird die Mèche, am 8. VI. die Nähte entfernt. Die Schmerzanfälle sind vollkommen verschwunden. Im Bereich des Trigeminus ist vollständige Anästhesie vorhanden, und auch die Geschmacks- 
und Geruchsempfindung ist aufgehoben. Vor allem zeigt aber das linke Auge schwere Veränderungen, so daß Patientin in die Augenklinik transportiert werden muß.

Dort wird konstatiert: Großes Korneainfiltrat, Hypopyon der vorderen Kammer, Iritis hohen Grades, Kornea und übriger Bulbus ohne Sensibilität. Parese des Orbicularis. Keratitis lagophtalmo et neuroparalytica. Es tritt ein Prolaps der Iris nach Perforation des Ulcus ein und Patientin wird nach 6 Wochen gegen ärztlichen Rat mit Phthisis bulbi incipiens entlassen.

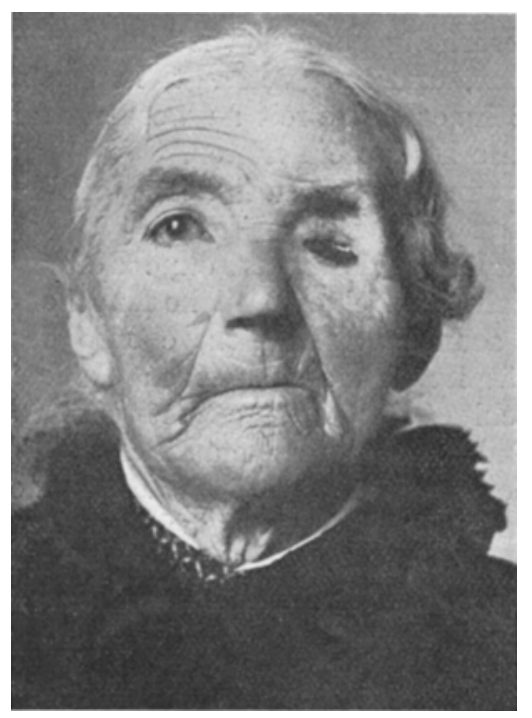

Fig. I.

Nach 2 Monaten neuerdings Fintritt. Staphyloma, totale Corneae, stark vorgetrieben. Bulbus auf Berührung völlig unempfindlich. $\mathrm{T},=+\mathrm{r}$. Glaucoma secundarium. Rechts Cataracta senilis incipiens. Am I8. Juli I9ro ohne jede Empfindung verlaufend Enucleatio bulbi, Catgutnaht. Reizloser Verlauf. Am 29. August entlassen (Fig. I).

R. J., 30 jährige Jungfrau von Staretschwil. Erster Eintritt I4. IV. Ig08. Mutter starb an unbekannter Krankheit. Vater lebt, 78 Jahre alt, und ist gesund. Eine Schwester starb vor 25 Jahren an Tuberkulose, eine Schwester ist unterleibsleidend. Als Kind ist Patientin schwer krank gewesen (Ohrenschmerzen und AusfluB) und hat schon in der Schule an Schwindelanfällen gelitten, die im I3-- I7. Lebensjahre fast wöchentlich auftraten.

Zur Zeit der Periode, die mit 20 Jahren einsetzte, sind die Schwindelanfälle selten. Die Periode war stets unregelmäßig mit 6-8 Wochen Intervall, schwach 2 Tage dauernd. Bevor die Periode einsetzte, sollen sich die Schwindelanfälle gehäuft haben. Mit 23 Jahren litt Patientin an Magenschmerzen krampfartiger Natur, bei denen Brechen vorhanden war, doch war kein Blut im Erbrochenen vorhanden. Mit 26 Jahren hörte das Erbrechen langsam auf, ist jedoch jetzt noch zeitweise vorhanden.

Im Jahre rgo3 sollen plötzlich ohne eine Ursache heftige Schmerzen unter und über dem rechten Auge aufgetreten sein, die sich dann auch auf den rechten Gaumen und die rechte Nase erstreckten. Seit dieser 
Zeit treten diese Anfälle intermittierend auf, so daß pro Tag 4-Io Anfälle von Io-20 Minuten Dauer vorhanden sind. Im Herbste und Winter sollen die Anfälle stärker als im Sommer sein.

$S$ t a $t$ u s: Ordentlich genährte Person. Herz und Lungen ohne Besonderheit. Die Austrittspunkte des rechten Supra- und Infraorbitalis sind druckempfindlich. Das Gebiet dieser Nerven zeigt eine deutliche Hyperästhesie. Das rechte Lid etwas ödematös und leicht gerötet. Die Lidspalte ist rechts kleiner als links. Die Pupillen sind beiderseits gleichweit. Facialis o. B. intakt. Während des Schmerzanfalles ist rechts eine lebhafte Tränenabsonderung vorhanden. Die rechte Hälfte der Gaumenbogen und der Uvula sind deutlich gerötet. Die Rötung ist während des Anfalles intensiver und in der Mittellinie scharf abgesetzt. Nach dem Anfalle geht die Rötung sukzessive zurïck und ist nicht mehr so scharf und deutlich alszugrenzen. Die Schmerzen erstrecken sich bis in die Gebiete des weichen und harten Gaumens.

Urin: ohne Befund. D i a g nos e: Neuralgie Trigeminus I und II.

Therapie: Elektrisieren mit Salusapparat, erfolglos.

30. IV. Operation in rtlhiger $\mathrm{MoCl}_{3}$-Narkose (Dir. Dr. H. Bircher). Neuraxeresie nach $T$ hiersch, des Nerv. supra- und infraorbitalis, was gut gelingt.

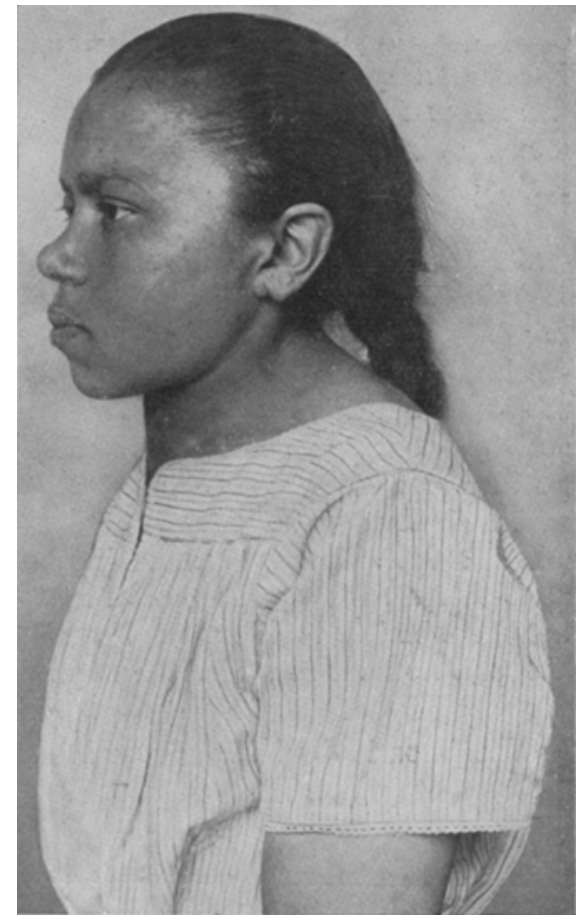

Fig. 2.

3I. IV. Die Gebiete des Nv. infra- und supraorbitalis werden anästhetisch. Die Anfälle vermindern sich an Zahl und Intensität, so daß Patientin am 24. V. Igo8 gebessert entlassen werden konnte. In der Folgezeit litt Patientin etwas weniger unter den heftigen Schmerzen, die aber im Winter I909-IgIo an Intensität und Zahl zunahmen und im März igio laut Aussagen des einweisenden Arztes, Dr. Sulser in Mellingen, folgendes qualvolle Bild machten: Die Schmerzen treten am heftigsten in der rechten Hälfte der Nase auf, beherrschen fast die 
ganze Gesichtshälfte und z. T. den Hinterkopf, die Zähne. Sie werden äußerst heftig, durch Kau- und Schluckbewegungen ausgelöst. Patientin ist in letzter Zeit etwas schwermütig geworden und das angestrengte Arbeiten fiel ihr sehr schwer.

$\mathrm{S}$ t a t us (Priv.-Doz. Dr. B ing und Dr. Eugen B ir cher).

Sehr gut genährte kräftige Person (Fig. 2 und 3). Deutlich mongoloider Gesichtstypus. Die Lippen sind gewulstet, der Nasenrüchen eingezogen. Eine Schwellung der rechten Gesichtshälfte ist sehr auffallend, die bis zu den

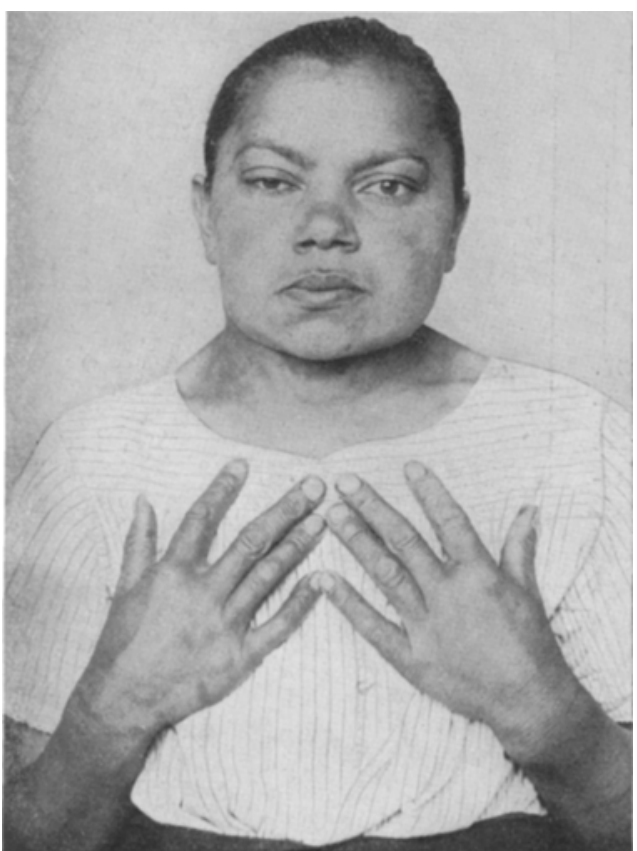

Fig. 3 . Augenbrauen hinaufreicht. Fingerabdrücke könren nicht hervorgerufen werden. Die Temperatur der rechten Wange scheint etwas erhöht $\mathrm{zu}$ sein. Von Asymmetrien beim Schwitzen weiß Patientin nichts anzugeben.

Im Gebiete des Schädeldaches ist eine deutliche Assymmetrie wahrzunehmen, indem rechterseits am Scheitelbein eine deutliche Abflachung nachzuweisen ist.

Die Haare sind dünn, trocken, zeigen keine Richtungsanomalien.

Die rechte Iidspalte ist gegenüber der linken verengt, so da $\beta$ das Oberlid beim Blick geradeaus die Pupille halbiert. Eine deutliche Parese im Levator palpebrae ist objektiv nicht festzustellen. Rechts ist ein deutlicher Enophtalmus, während links mäßiger Exophtalmus vorhanden ist.

A u g e n bef und: Oberarzt Dr. Vogt: Pupillarreaktion auf Licht und Koncergenz sehr gut. Ophtalmoskopisch: Papillen und Gefäße des Augenhintergrundes ohne Besonderheiten. Leichte Hypermetropie $\left.\frac{\mathrm{Rs}}{\mathrm{Ls}}\right\}_{6 / 8} \mathrm{H} \quad 0,75$.

Gesichtsfelder völlig ohne Besonderheiten. Augenbewegungen sind intakt. Die rechte Conjunctiva ist wesentlich deutlicher injiziert als links. Patientin gibtan, daß, wenn sie jemand länger beobachtend fixiere, Doppeltsehen auftreten könne. 
Die auf den Vertex aufgesetzte Stimmgabel wird konstant rechts lateralisiert, $\mathrm{R}$ i n $\mathrm{n}$ e beiderseits positiv, I uftleitung beiderseits größer als Knochenleitung.

Die Zunge kann gerade vorgestreckt werden, ist leicht belegt, etwas zitternd, sicher nicht hypertrophisch. Der Masseterenreflex zeigt sich entschieden gesteigert.

Geschmackspr ï f ung: Tinct. Gentianae bitter. Glzyerin süß.

Alum. acetic. sauer. Kal. bromat. salzig.

O $1 \mathrm{factorius.} \mathrm{Aus} \mathrm{dem} \mathrm{Liquor.} \mathrm{Ammoni} \mathrm{assisal} \mathrm{wird} \mathrm{das}$ Anis herausgerochen, Ammoniak wird kaum gerochen. Essigsäure wird säuerlich angegeben, rechts deutlicher empfunden. Die Gaumenmotilität zeigt keine Besonderheiten. Auf der rechten Stirnseite ist eine deutliche Kältehyperästhesie vorhanden. Pinselberührung ist im ganzen Bereiche des Gesichts positiv, im zweiten Aste deutlich schwächer. Nadelspitze wird überall gleichmäßig empfunden. Über dem Proc. styloideus I6 1/2 rechts, I7 $1 / 2$ links.

Die Sehnenreflexe sind in den rechten oberen Extremitäten sehr leicht, links fast gar nicht auszulösen. Beriihrungs-, Vibriations- und Schmerzempfindung sind an den oberen Extremitäten vollständig gleich, der Spannungszustand ebenfalls. An der unteren Extremität sind die Reflexe lebhafter als links.

Die rechte Hand und der rechte Vorderarm sind etwas geschwollen, so daß Fingerabdrücke bestehen bleiben. Die Schwellung geht hie und da jedoch sehr selten zurück. Im rechten Arme wie im rechten Beine leide sie hie und da an Schwellungen. Die Hand im dulabu groß.

Der Hals ist kurz. Die Venen sind stark geschwollen. In der Mitte ist eine rundliche Struma von Borsdorferapfelgröße nachweisbar. $38 \mathrm{~cm}$ Umfang. Stuhl und Urin ohne Bescnderheiten. Röntgenaufnahmen des Schädels und der Extremitäten zeigen keine Besonderheiten.

Während der Aufnahme des Status sind einige Schmerzanfälle eingetreten. Zuckungen im rechten Orbicularis oculi sind während des Anfalles deutlich vorhanden. Der Puls ist klein, 92, während er außerhalb des Anfalles wesentlich kräftiger ist. Die Dauer des Anfalles heträgt $2 \frac{1}{2}$ Minuten. Die Anfälle folgen sich in Intervallen von ca. Io Minuten.

3. IV. I9Io. Halbsitzende Stellung. Mo. $\mathrm{CHCl}_{3}$-Narkose $\mathrm{R}$ o th Draeger. Ganglionexstirpation nach Krause-Leer (Operateur: Sekundärarzt D r. E u g e n B ir c h e r).

I. Schnitt am Vorderrande des Sternocleidomastoideus, die Carotis externa wird in typischer Weise freigelegt und unterbunden.

2. Lappenschnitt nach $\mathrm{Kr}$ a u s e am Ohr beginnend, im Bogen nach vorne bis zum Jochbeinansatz ziehend. Das Jochbein wird temporär durchtrennt, die Temporalmuskulatur stumpf weggeschoben, 
das Periost gelöst und nun mit der Doyenschen Fräse die Temporalgegend eröffnet und ein ca. 5 frankstückgroßes Knochenstück mit der Fräse herausgesägt. Das Gehirn, insbesondere die Dura, ist kräftig gespannt, und es ist ausgeschlossen, von dieser Offnung aus an das Ganglion Gasseri zu gelangen, und unter Opferung des Knochens wird die Offnung nach oben sowie nach unten bis an die Umbiegungsstelle zur Schädelbasis erweitert, bis eine kleinhandtellergroße Offnung vorhanden ist.

Die Dura wird langsam von der Knochenbasis stumpf abpräpariert und dabei ohne weiteres die Meningea media durchtrennt. Hie und da müssen dabei Verwachsungen der Dura mit der Schädelbasis gelöst werden. Es gelingt nun mit großer Mühe, das Gehirn empor zu heben und auf einer flachen, mit Gaze umgebenen gynae kologischen Spatel zu halten, da es sehr stark gespannt ist. Bei der außerordentlichen Breite des Schädels macht es ebenfalls Schwierigkeiten, bis in die Tiefe zu gelangen was, nur mit Mühe gelingt. Nach langer Präparierarbeit, die regelmäßig durch Tamponade mit Adrenalintampon unterbrochen wird, gelingt es endlich, den dritten Ast am Foramen ovale frei zu bekommen, von diesem aus gelangt man rasch an das Ganglion, von hier zum zweiten Ast. Zweiter und dritter Ast werden am Foramen ovale bzw. rotundum scharf durchtrennt: beide Löcher durchstopft und das freipräparierte Ganglion mit einer Kornzange samt dem ersten Ast ausgerissen. Im Moment des Ausreißens tritt eine ganz erhebliche Blutung auf, die aus dem vorletzten Sinus cavernosus stammt, auf Adrenalintamponade jedoch steht, und auch bei der Revision des Wundgebietes, ob das ganze Ganglion entfernt sei, nur mäßig stark auftritt. Das stark und mit ziemlich großer Gewalt emporgehobene Gehirn wird in sein altes Bett zurückgelegt, eine feine Mèche eingelegt und exakte $W$ undnaht ausgeführt. Minute.

Der Puls war während des ganzen Eingriffes kräftig, $60-80^{\circ}$ pro

Nach der Operation Kochsalzinfusion. Digalen, Rektaleinläufe.

V e r l a u f: 4. IV. Die Anfälle haben prompt aufgehört, hingegen werden heftige Schmerzen im Hinterhaupt angegeben. Die Mèche wird entfernt, wobei sich etwas seröse, rötlich dingierte Flïssigkeit entleert. Geschmackssensibilität ist auf der Zunge verschwunden, im Gaumen erhalten.

9. IV. Immer noch Schmerzen im Hinterhaupt, suspeckte Occipitalneuralgie, Entfernung der Nähte, Heilung p. p.

I2. IV. Es zeigt, sich, daß Patientin seit der Operation im psychischen Verhalten vollkommen verändert ist. Verweigert zeitweise jegliche Auskunft, wird dann wieder sehr gesprächig, lacht ohne Grund laut auf, singt unmotiviert, teilweise obsköne Lieder Spricht wirres Zeug: Jetzt kommt meine Schwester mit einem Kübel Milch. Ich gehe morgen nach Baden in die Kur. Sie klagt über Zahnschmerzen, Schmer- 
zen in den Gliedern, im Gesäß usw., sie weigert sich, sich verbinden zu lassen.

26. IV. klagt sie wieder über heftige Occipitalschmerzen, die aber bald wieder verschwinden.

27. IV. Klagen über Magenschmerzen, die wieder verschwinden. Patientin wird unklar, betet eine Stunde lang für alle Sünden der Welt und betont, daß sie auch den sündigen Arzt in ihr Gebet einschließe.

30. IV. Patientin ist wiederum vollkommen klar, klagt etwas über seine Krämpfe, die sofort verschwinden.

IV. Plötzlich Temperatursteigerung auf $39^{\circ}$, ohne daß dafür ein Grund gefunden werden kann. Patientin ohne Beschwerden, wird klar.

20. V. Temperatur abgefallen.

Io. V. Entlassung.

3. IX. Status: Lokal usw. geheilte lappenförmige Narbe in der rechten Temporalgegend, die eine mäßige Eindellung zeigt. Auf Druck mäßig empfindlich, geringe Pulsation. Am 3I. X. ist die Narbe fest, etwas eingewolbt, doch hat sie vom Periost aus ein teilweise knöcherner Verschluß gebildet. (Fig. 4).

Die Schwellung auf der

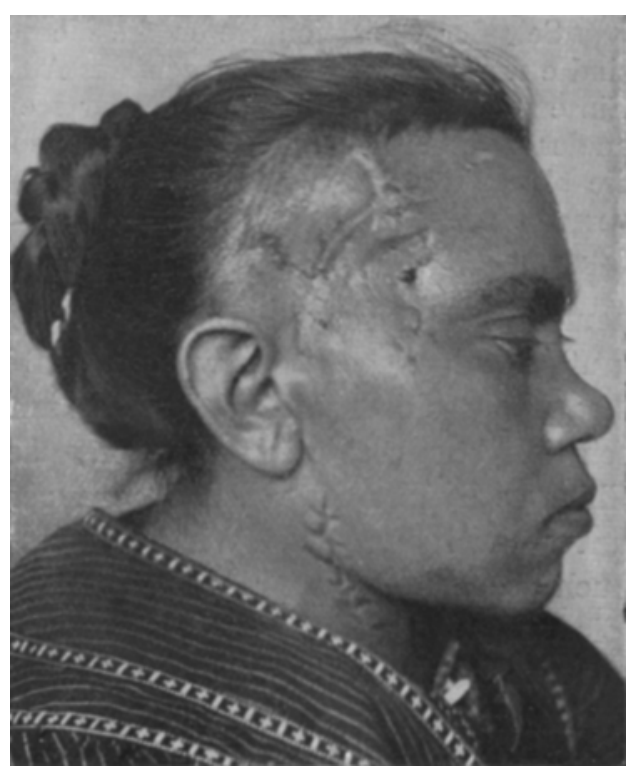
rechten Seite ist zurück-

Fig. 4. gegangen, so daß keine Fingerabdrücke mehr vorhanden sind, der Liderspalt ist beiderseits gleichweit. Temperatur beiderseits dieselbe. Die Zunge weicht etwas nach rechts $a b$, ist etwas belegt. Der Gaumen zeigt keine besondere Rötung mehr. Der Masseterenreflex ist erloschen. Die Korneal- und Palpebralreflexe fehlen rechts. Die Funktion des Levatorpalpebrae ist erhalten. Im ganzen Bereich der Trigeminus ist eine vollkommene Anästhesie. Die Pupillenresektion beiderseits prompt vorhanden.

$\begin{array}{rrr}\text { Geschmacksprüfung: Zunge: sauer } & - & \text { negativ } \\ \text { salzig } & - & , \\ \text { süß } & - & , \\ \text { bitter } & - & ,\end{array}$


Geruch: Kampfer stark herabgesetzt

Ammoniak stark herabgesetzt.

Links sehr deutlich vorhanden.

A u g e: Äußere Augenmuskeln intakt. Lähmung des Abducens rechts, wodurch Doppelbilder entstehen. Papillarreaktion auf Akkomodation und Konvergenz prompt, ebenso auf Licht. Pupillen mittelweit.

Beidseits leichte Hypermetropie, sonst ohne Besonderheiten.

Patientin hat keine Anfälle mehr gehabt, kann arbeiten, nur daß das Auge hie und da etwas Beschwerden macht.

Seit der Operation treten die Menses regulär alle 4 Wochen auf, sind etwas schwach und zeigen das Auffallende eines vikarisierenden Einsetzens, indem sich zur Zeit der Menses an beiden Oberschenkeln an der Innenseite blutunterlaufene Stellen bis zu Handtellergröße bilden, die blau, grün, dann gelb werden und bis zur nächsten Menses völlig verschwinden.

Beide Fälle sind durch die Operation von der Trigeminusneuralgie völlig geheilt. Dieses Resultat war zu erwarten, immerhin muß darauf hingewiesen werden, daß es sich nach unsern Kenntnissen der chirurgischen Technik um einen der technisch allerschwierigsten Eingriffe handelt, an den mehrere namhafte Chirurgen nicht ohne Scheu gegangen sind, andere ihn nicht zu unternehmen wagten. Wir halten es für absolut notwendig, wie auch schon $\mathrm{K}$ och e $\mathrm{r} \mathrm{u}$. a. darauf hingewiesen haben, daß der Eingriff an der Leiche geübt werden muß, so daß man über die topographisch-chirurgische Anatomie des ganzen Gebietes genau orientiert ist. Dann tut man gut daran, den Rat $\mathrm{K} \mathrm{r}$ a u s e s zu befolgen, und während der Operation ein gutes Schädelnervenpräparat zur Hand zu haben, an dem man sich jederzeit über die Lage der Organe klar werden kann.

Daß man auf das Alter der Patienten keine Rücksicht zu nehmen braucht, beweist unsere erste Beobachtung, die nach dem Falle von Tiffany (79 Jahre), mit 77 Jahren die älteste operierte Patientin darstellt. Während der zweite der von mir operierten Fälle neben den 24 Jahre alten Patienten von Gutierrez eine der jüngsten Beobachtungen darstellt.

Daß man einen derartigen schweren Eingriff nur bei ganz strengen Indikationen vornehmen darf, muß als selbstverständlich erachtet werden. In der ersten Beobachtung war die Indikation zum intrakraniellen Eingriff gegeben. Nachdem im Verlaufe von 
If Jahren $7 \mathrm{mal}$ peripher operiert worden war, und sozusagen jedesmal ein Erfolg für gewisse Zeit zu erzielen war, so wäre es von vornherein nutzlos gewesen; die Zeit mit einem extrakraniellen Eingriff an der Basis zu vergeuden. Die Regenerationsfähigkeit der Nerven nach 7 Operationen hatte sich zur Genüge erwiesen, so daß auch nach einer Resektion des 2. und 3. Astes an der Basis sicher mit einer Rezidive zu rechnen gewesen wäre. Um so eher wurde man aber zu dem intrakraniellen Eingriffe gezwungen, da auch der I. Ast von der Neuralgie befallen war. Wer 7 mal die Nervenausreißung mitgemacht hat, wobei mehrmals längere Nervenstücke entfernt worden sind, den sollte man nicht länger mit den peripheren Operationen quälen. Es ist gewiß nicht nötig, noch häufiger peripher zu operieren, wie in dem Falle von $\mathrm{Busch}-\mathrm{Tren-}$ delenburg, der von I879-I892 23 periphere Operationen durchmachte und nach 92 noch wiederholt operiert worden sei.

In der zweiten jüngeren Beobachtung war es schon schwieriger, die Indikation zu stellen, ob man sofort zur intrakraniellen Operation schreiten solle. Vor allem war für uns maßgebend, daß alle drei Trigeminusäste, speziell der erste, am heftigsten von der Neuralgie befallen waren. Im weitern sprach der ganze Verlauf der Affektion, der Typus der Schmerzanfälle, für einen intrakraniellen Sitz der Affektion. Auch $\mathrm{H}$ ulles empfiehlt neuerdings beim Ergriffensein aller drei Äste sofort intrakraniell vorzugehen. Schon beim ersten Spitalaufenthalte zeigten sich alle drei Äste von der Affektion erfaßt, die starke Thränensekretion spontaner Natur wies auf die Beteiligung des Lakrimalanteil des I. Astes hin, wahrscheinlich dürfte auch der Facialisanteil der Glandulalacrimalis dabei beteiligt gewesen sein, was ebenfalls auf eine zentrale Läsion in der Gegend des Ganglion hindeuten würde; die intensive Thränenabsonderung während der Schmerzattacken läßt auf eine starke Reizung der Supraorbitalis schließen.

Ebenso wichtig schien uns in diesem Falle die Beteiligung vasomotorischer Nerven des sympathischen Nervensystems zu sein, die sich durch Rötung der Gaumenbogen und Uvula, durch Lidödem, durch Schwellung und Temperaturerhöhung der ganzen rechten Gesichtshälfte kennzeichnete. Der ausgesprochene rechtsseitige Enophtalmus dürfte ebenfalls damit in Verbindung $\mathrm{zu}$ bringen seir. 
Diese Tatsache weist den Sitz der Erkrankung von vornherein zentralwärts, und zwar entweder in das Ganglion ciliare, sphenopalatium und oticum oder weiter zentralwärts in das Ganglion Gasseri. Die nachgewiesene Lidspaltenverengerung und der Enophtalmus deuteten darauf hin, daß die durch die obenbezeichneten Ganglien verlaufenden sympathischen Fasern ad musculum tarsalem superiorem, ad musculum orbitalem ad vasa sanguinea eine Veränderung in lädierendem Sinne erlitten hatten, und diese Läsion mußte zentralwärts gesucht werden.

Aber nicht nur die die Trigeminusäste begleitenden Sympathicusfasern weisen auf dessen Mitbeteiligung, sondern wir sehen auch in diesem Falle, daB vasomotorische Störungen sich im Bereiche des rechten Armes Reflexerhöhungen in den Extremitäten der rechten Seite geltend machten. Ebenso unzweifelhaft war während der Anfälle eine deutliche Verengerung der Pulsqualität und Zunahme der Frequenz, also eine vasomotorische Störung nachweisbar. Alle diese Punkte deuten auf eine mehr zentralwärts gelegene Erkrankung hin, und es ist nicht ausgeschlossen, daß die vasomotorischen Erscheinungen im rechten Arme direkt von Erkrankung des Ganglion Gasseri auf sympathischem Wege weitergefüht herrüren.

Daß sympathische Fasern bei der Trigeminusneuralgie beteiligt sein müssen, darauf weisen auch die trophischen Störungen an den Gesichtsknochen hin, die nach der peripheren Ausreißung der Nervenstämme aufgetreten sind.

Das radikalere intrakranielle Vorgehen war in diesem Falle ebenso unzweifelhaft gegeben wie in der ersten Beobachtung.

Bei der Operation hielten wir uns im allgemeinen an das $\mathrm{K} r$ a u s e sche Verfahren; machten uns aber auch die technischen Ratschläge zunutze, die von $\mathrm{L}$ e $\mathrm{x}$ e r und $\mathrm{Fridrich}$ gegeben worden sind.

In beiden Fällen nahmen wir die präliminäre Unterbindung der Carotis externa vor, wie sie von Fridrich empfohlen worden ist. Diese Unterbindung erleichtert das weitere operative Vorgehen außerordentlich, indem einer der schwierigsten Akte des Eingriffes, die Unterbindung der A. meningea media, dadurch umgangen wird. Von der temporären Unterbindung der Carotis externa, wie sie $z$. B. von $\mathrm{Gr}$ a v e s empfohlen wurde, glauben wir 
absehen zu können, da sie die Schwierigkeiten der Meningeaunterbindung nur wenig erleichtert, aber nicht vollkommen umgeht.

Schädlichkeiten von seiten der Unterbindung der Externa glauben wir keine gesehen zu haben, es sei denn, man wolle die einsetzende postoperative Psychose des zweiten Falles ihr zur Last legen. Die Psychose kann jedoch viel eher als von dem recht erheblichen Spateldruck herrührend betrachtet werden.

Der Unterbindung der Carotis externa muß in heilendem Sinne eine entschiedene Wirkung zugesprochen werden. Nicht wenig Fälle von Trigeminusneuralgien sind früher durch Kompression der Carotis communis in günstigem. Sinne beeinflußt worden. Hutchinson und Fowler konnten in ihren Zusammenstellungen über eine ganze Anzahl von Beobachtungen berichten, bei denen die Carotisunterbindung, wie sie von $\mathrm{NuB}$ b a u I I862 zum ersten Male ausgeführt wurde, gute Erfolge teilweise dauernder Natur zeitigte. $\mathrm{R}$ o s e $\mathrm{r}$ hat die Carotis externa bei Gesichtsneuralgien mit günstigem Erfolge unterbunden. Den Einfluß der Unterbindung der Carotis auf eine Läsion der in der Nähe verlaufenden sympathischen Fasern zurückzuführen, dürfte naheliegend sein.

Den Hautmuskelschnitt haben wir nach der Angabe Krauses ausgeführt; die Gefahr einer Verletzung der Stirnfacialisäste ist bei diesem Schnitte unbedingt vorhanden, und in einem Falle haben wir die Lähmung des Stirnfacialis auch auftreten sehen. Wir möchten daher für die Zukunft den $\mathrm{Koch}$ erschen nach unten konvexen Schnitt vorziehen, der uns bei der Operation eines retrobulbären Tumors mit Sicherheit die Facialisäste vermeiden ließ.

Eine wesentliche Erleichterung des späteren Vorgehens ist der temporären Keilbeinresektion zuzuerkennen, die sich mit einigen Meißelschlägen leicht vornehmen läßt. Ebenso ist dem von $\mathrm{L}$ ex e r empfohlenen und von $\mathrm{K}$ och e $\mathrm{r}$ bestätigten Vorschlag, ,sich bei der Trepanation nicht auf ein osteoplastisches Verfahren zu versteifen", sondern den Knochen wegzunehmen bis zur Crista und auch von der Basis den Knochen nach Lösung des Periostes zu entfernen, zuzustimmen. Daß man gerade bis an das Foramen spinosum mit der Entfernung des Knochens gehen 
müsse, halten wir für überflüssig. Die Entfernung von Knochenpartien an der Basis schafft sowohl für die Orientierung als auch für die Hebung des Gehirns mit dem Spatel wesentlich günstigere Verhältnisse.

Ist die Carotis externa unterbunden, so läßt sich das Gehirn mit der Dura leicht und rasch abschieben, und man gelangt sicher zu dem ins Foramen ovale verlaufenden 3. Ast. Ist dieser aufgefunden, so gelangt man durch vorsichtiges Präparieren leicht an das Ganglion, das soweit als die Blutung es gestattet, von der Unterlage und der Dura abzupräparieren ist. Mit einer gut fassenden Zange kann es nach Durchschneidung des 2. und 3. Astes extrahiert werden. Die Foramina ovale und rotundum werden mit einer Sonde am sichersten noch ausgestopft.

Der letzte Moment, die Entfernung des Ganglions, ist an $\mathrm{Zu}$ fällen der reichste, gewöhnlich wird der Sinus cavernosus verletzt, was zu einer recht erheblichen Blutung führt, wie sie in unserer zweiten Beobachtung auftrat. Die Blutung läßt sich durch Tamponade, ich glaube in unserem Falle hat das Adrenalin gute Dienste geleistet, rasch zur Stillung bewegen. Bei der Ausdrehung, wenn man mit der Zange etwas medial gelangt, liegt die Gefahr einer Abduzensverletzung ebenfalls nahe, wie sie in unserer zweiten Beobachtung passiert ist. Die Blutung aus den kleinen Venen verlangt eine feste Tamponade und auch dabei kann der Abducens verletzt werden. Die Blutung kann so groß werden, daß, wie T ürk angibt, in Io Fällen die Operation völlig unterbrochen werden mußte. Auch beim Freipräparieren des Ganglions ist eine Verletzung nicht ausgeschlossen. L e $\mathrm{x}$ e $\mathrm{r}$ berichtet unter seinen I5 Fällen, daß 5 mal Abducenslähmung eingetreten sei, die teilweise im Laufe der Zeit zurückging, auch in unserer Beobachtung ist eine Besserung der Abduzenslähmung zu konstatieren gewesen. Neben diesen Gefahren drohen bei der Operation von seiten des Spateldruckes auf das Gehirn ungünstige Einflüsse. K r a u s e beobachtete infolge des Spateldruckes aphasische Stauungen in drei Fällen, Gutierrez halbseitige Lähmung, P o p p e r t sogar einen Todesfall durch Hemiplegie. Von B e ck, Gers te r u.a. konnten Erweichungsherde und Abszesse später im Temporallappen festgestellt werden, und auch $\mathrm{L}$ e x e r berichtet von ähnlichen Zuständen. 
Während in unserer ersten Beobachtung das durch das Aiter schon etwas atrophisch gewordene Gehirn sich leicht emporheben ließ, so war dies in der zweiten Beobachtung mit großen Schwierigkeiten verknüpft. Gerade bei mehr jugendlichen Personen, bei denen das Gehirn einen größeren Turgor aufweist, ist der Spateldruck sehr zu fürchten. Die von uns beobachtete postoperative Psychose möchten wir unbedingt auf den Spateldruck zurückführen. Die Störungen können spontan sich vollständig zurückbilden.

Neben diesen bald nach der Operation auftretenden Störungen ist kurz der später einsetzenden Affektionen zu gedenken. Von fast allen Operateuren, die über mehrere Fälle von Ganglionexstirpationen berichten konnten, wird am meisten über Augenstörungen berichtet. In 30 Fällen ist nach $\mathrm{T} \ddot{\mathrm{u}} \mathrm{r} \mathrm{k}$ Conjunctivitis Ulcus corneae, Keratitis oder Hypopyon aufgetreten; in 2 Fällen trat Erblindung ein, während in 4 Fällen wegen Phthisis bulbi die Enukleation vorgenommen werden muBte. Diesen 4 Fällen reiht sich unsere Beobachtung als fünfte an. Während in unsern beiden Fällen ganz dieselbe Nachbehandlung mit Uhrglas über das Auge nach den Vorschriften von $\mathrm{Kr}$ a $\mathbf{u}$ s e stathatte, so traten in dem alten der beiden Fälle diese üblen Nachwirkungen auf. Wir glauben nicht, daß in unserem Falle die Enukleation des Auges allein auf die Ganglionentfernung zurückgeführt werden darf, denn die zahlreichen peripheren infra- und supraorbitalen Nervenresektionen hatten zu einem schweren Ektropium geführt, das einen Lagophthalmus nach sich zog, der zu Keratitis und dem Hypopyon führte. Die Entfernung des Ganglions dürfte die Phthisis bulbi nur beschleunigt haben.

Sind die Resultate der Ganglionexstirpationen, da sie regelmäßig mit unerwünschten Ausfallserscheinungen verbunden sind, keine idealen, so sind sie unzweifelhaft in der Heilung des schweren Trigeminusleidens die zuverlässigsten.

Durch van Gehuchten, Spiller und Frazier, neuerdings durch $\mathrm{R}$ a s m ow s $\mathrm{y}$, ist versucht worden, eine Besserung der Resultate dadurch zu erzielen, daß sie von der Exstirpation des Ganglions absahen und sich damit begnügten, die zentrale Wurzel des Ganglions zu durchtrennen. Die Gefahr der Blutung wird auf diese Weise auf ein Minimum reduziert; 
ebenso die Nebenverletzungen des Oculomotorius, Trochlearis und Abducens können leicht vermieden werden.

Am wichtigsten erscheint es jedoch, da $B$ keine trophischen Störungen speziell von seiten des Auges auftreten, indem die dem I. Trigeminus sich beimischenden Sympathikusfasern nicht lädiert werden. Spiller und Frazier, wie auch Dollinger und Rasumowsky haben mit dieser sogenannten "phynologischen Exstirpation des Ganglion Gasseri nach van Gehuchten in einer Reihe von Fällen (ca. 40) gute Erfolge aufzuweisen, hingegen ist über die Dauerresultate nur wenig bekannt geworden. Und nur Fra z i e r verfügt über Dauerheilungen bis zu 7 Jahren.

Bevor eine größere Anzahl von Dauerheilungen mit dieser Methode erzielt worden, tut man gut, vorläufig das für die Dauerheilung sichere Verfahren von $\mathrm{H}$ a $\mathrm{r} \mathrm{l}$ e $\mathrm{y}-\mathrm{Kr}$ a u s e zu wählen.

In unsern beiden Beobachtungen kann die Operation quoad sanationem als gelungen bezeichnet werden. Es ist neurologisch interessant zu konstatieren, daB alle die auf die Sympathikusbeteiligung zurückführenden Symptome (Exophthalmus, Asymmetrie der Lidspalte, vasomotorische Stauungen) durch die Operation vollständig behoben werden sind. Die ganze Trigeminuspartie vom I., 2. und 3. Ast ist vollständig anästhetisch, der Niesreflex konnte nicht mehr auf der operierten Seite ausgelöst werden; die stechenden Sensationen von Ammoniak und Essigsäure konnten nicht mehr gefühlt werden.

Ebenso zeigten sich die vom Ganglion sphenopalatinum durch den Ramus II im Ganglion Gasseri führenden Geschmacksfasern, welche via nervus petrosus superficialis major zum Ganglion geniculi und dem Facialis und von da durch die Chorda tympani zum Lingualis und den vordern zwei Dritteln der Zunge gelangen, für alle Geschmackssensationen bis zur Stunde vollkommen anästhetisch. Eine Monoplegia masticatoria ist ebenfalls vorhanden. Die Salivation ist nur gering verändert, was zu erwarten war.

Die Ausfallserscheinungen nach der Operation sind die beste Kontrolle, wie radikal man in der Entfernung des Ganglion und Gasseri und seiner Äste gegangen ist.

Wird die Operation nach genauer Untersuchung und strenger Indikation ausgeführt, so ist sie imstande, großes zu leisten und Schwerleidenden die einzig sichere Hilfe zu bringen. 


\section{$\mathrm{Nachtrag}$.}

Nachdem vorliegende Arbeit schon in Druck gegangen war, hatten wir Gelegenheit, einen weitern Fall von Trigeminusneuralgie nach dem $\mathrm{Kr}$ a us e- $\mathrm{H}$ a r t le y schen Verfahren $\mathrm{zu}$ operieren. Wir möchten nicht versäumen, diesen Fall den beiden übrigen beizufügen, obschon er nicht so glücklich verlaufen ist. Aber gerade wegen des dabei erhobenen Sektionsbefundes kann er auf ein gewisses Interesse Anspruch erheben.

3. Der im Jahre 1840 geborene Knecht B. N. erkrankte im Jahre I906 an heftigen Schmerzen im linken Unterkiefer, die sich besonders bei Nahrungsaufnahme zu einer solchen Höhe steigerten, daß er häufig das Essen aussetzen mußte. Er will schon früher an ähnlichen Schmerzen gelitten haben. Aus der Familienannamnese ist zu entnehmen, daß der Patient dem Alkohol und dem übermäßigen Tabakgenuß ergeben war.

Patient wird am I3. IX. I907 der Anstalt zugewiesen. St a t us: Kr̈äftiger alter Mann, Herz und Lunge ohne Besonderheiten. Der linke Unterkiefer wie auch die ist auf geringe Berührung heftig druckempfindlich. Das Infraorbitalis ist wenig druckempfindlich, der Supraorbitalis schmerzfrei. Medikamentöse und elektrische Therapie in verschiedenster Applikationsweise bleibt erfolglos.

Am 30. IX. werden der N. infraorbitalis an seinem Austritte und der Mandibularis am Kopfwinkel ausgerissen. Es tritt Wundheilung ein. Patient wird am 20. X. I907 geheilt entlassen.

Nach ca. 6 Monaten Heilungsdauer setzt die Neuralgie von neuem mit großer Heftigkeit ein und Patient läßt sich verschiedentlich auch mit Schlösserschen Alkoholinjektionen behandeln, ohne daß dadurch eine Besserung eingetreten wäre. Daher am 3I. X. Igro neuterdings Spitaleintritt.

S t a t u s: Kräftiger, alter Mann. Mäßige Tortdicollis nach rechts. Die ganze linke Gesichtshälfte ist schräg verzogen, die Muskulatur, speziell die Kaumuskulatur, ist atrophisch. Ebenso scheint Knochenatrophie vorhanden zu sein. Die Reflexe sind etwas erhöht. Pupillarund Kornealreflexe normal. Im Bereiche des zweiten und dritten Trigeminusast ist eine deutliche Hyperästhesie und Hyperthermie nachweisbar. Die Tränensekretion links ist etwas vermehrt. Auf Druck wird der zweite und dritte Trigeminusast heftig druckempfindlich. Es können auf diese Weise sogar einmal neuralgiforme Anfälle ausgelöst werden. Die Schleimhaut der linken Mundhälfte zeigt eine auffallende Rötung gegenüber rechts und ist sehr empfindlich auf Berührung. Sämtliche Geschmacksqualitäten auf den vorderen zwei Dritteln der Zunge sind sozusagen fast aufgehoben, wenigstens hochgradig herabgesetzt, ebenso zeigen sich die Geruchsqualitäten für Anis und Ammoniak rermindert. 
Während die Anfälle in der ersten Zeit des Krankenhausaufenthaltes sich alle Stunden 2-3 mal folgten, wobei das Gesicht auf der linken Seite krampfhaft zusammengezogen wurden, traten sie in den letzten Wochen fast von $3 \mathrm{zu} 3$ Minuten auf und wurden immer heftiger, so daß Patient nach erfolgloser Elektro- und Medikotherapie selbst die Operation vorschlug.

Herz nach links verbreitert. Die Temporalarterie geschlängelt, kaum comprimierbar: Puls 70 ; kräftig.

Operation am I4. XII. I9Io. (Operateur: Sekundärarzt Dr. E. B ircher) in ruhiger Mo. - $\mathrm{CHCl}_{3}$-Narkose mit Roth-DraegerApparat.

Praeliminäre Unterbindun gder Carotis intera, welche kleinfingerdick, stark arteriosklerotisch verändert ist.

Hautschnitt nach $\mathrm{K}$ o c h e r am vorderen des Proc. zygomaticus beginnend, am hinteren Ende gegen die Schläfe schräg aufsteigend.

Der Processus zygomaticus wird osteoplastisch reseziert, der Musc. temporalis vom Knochen nach vorne abgeschoben, mit der Doyenund Sudeckschen Fräse der gut $4 \mathrm{~mm}$ dicke Schädelknochen auf $4: 4 \mathrm{~cm}$ eröffnet und das Knotenstück entfernt. Mit der Knochenzange wird ein Teil des Bodens der mittleren Schädelgrube entfernt. Bei der Lösung der Dura vom Knochen entsteht ein $2 \mathrm{~cm}$ langer Durariß, woraus sich etwas Liquor entleert, worauf sich das prall gespannte Gehirn etwas leichter in die Höhe halten läßt. Während der I.ösung der Dura tritt eine gewaltige venöse Blutung auf, die nur durch eine längere Tamponade gestillt werden kann. Es zeigt sich, daß der Sinus petrosus superior bei der Lösung der Gehirnhaut verletzt wurde und daß die heftige Blutung aus dieser Öfnung stammt. Beim Weiterarbeiten in die Tiefe muß immer wieder gegen neı auftretende venöse Blutungen gekämpft werden, die auf eine längere Tamponade stets eintreten, aber das Vorgehen wesentlich verzögern. Auf diese Weise gelingt es nach langer mühevoller Arbeit, den dritten und zweiten Ast freizulegen, zu durchtrennen, beide an ihrem Abgang vom Ganglion Gasseri zu fassen und das Ganze auszureißen. Sofort wird der Gehirnspatel entfernt, das Blut ausgewischt. Ein Mèchedrain in den hinteren Wundwinkel eingelegt und die Wunde geschlossen. Dauer der Operation I Stunde 30 Minuten.

2 Stunden post op. setzt beim Patienten stertoröses Atmen ein, der Puls steigt auf I20. Patient ist aus der Narkose nicht erwacht.

6 Stunden post op. Temperatur 40,4. Puls kräftig, I20. Atmung stertorös 20. Patient läßt unter sich gehen. Pupillen eng, reagieren nur wenig. Sensibilität und Motilität vollkommen aufgehoben. Patellarreflexe vorhanden. Der durchblutete Verband wird gewechselt.

Io Stunden post op. Temperatur 4I,o. Puls kräftig, I 4o. Läßt unter sich gehen, sonst ostatus idem.

I4 Stunden post. op. Puls 8o. Atmung 24, oberflächlich. Pupillen- 
reaktion völlig aufgehoben, eng; Patellarreflexe schwach vorhanden. Sensibilität und Motilität völlig verschwunden.

$\mathrm{Da}$ Gehirndruck vermutet wird, wird die Wunde revidiert und eröffnet. $50 \mathrm{~g}$ coagulierten Blutes, welches sich an den Schädelwänden epidural befindet, werden entfernt, ein kleines, spritzendes Duragefä $\beta$ unterbunden und ein breites Gummidrain angelegt. Tamponade der Hautwunde. Kochsalzinfusion.

Der Zustand erfährt eine momentane Besserung; die Atmung wird

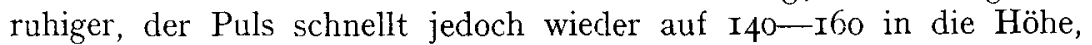
wird zusehends unregelmäßig und schwächer. Die Atmung wird stertorös, Cheyne-Stockes tritt auf: Die Pupillen werden eng. Nach und nach tritt tiefes Coma ein, in dem Patient 3 I Stunden post. op. stirbt.

Sektionsbefund: In der Operationswunde findet sich epidural wenig geronnenes Blut. Der linke Schläfenlappen ist von einer I mm dicken Schicht von geronnenem Blute bedeckt. Im Gyrus temporalis inferior und fusiformis fanden sich zahlreiche punktförmige Blutungen und ein ca. haselnußgroßer Erweichungsherd. Im Thalamus opticus sin. vornehmlich im Nucleus lateralis und in die Pars occipitalis der Cajsula interna ist ein stark nußgroßer, frischer, durchbluteter Erweichungsherd rorhanden. Das linke Ganglion Gasseri fehlt mit Ausnahme des Ramus primus, der vollkommen erhalten ist, ebenso des Abduceus. Im Sinus petrosus superficialis findet sich ein stark $\mathrm{Imm}$ langer Riß. Die Gehirnarterien sind alle stark erweicht, vergrößert, geschlängelt und ihr Lumen ist mit atheromatösen Massen angefüllt. Das übrige Gehirn ohne Besonderheiten.

Das Herz myodegeneriert; Coronarsklerose, Sklerose der Aorta. Schrumpfniere. Linksseitige Pleuritis und Bronchopneumonie des linken Unter- und Mittellappens.

Vorliegende Beobachtung ergänzt meine ersten beiden Fälle in interessanter Weise. Der zojährige Patient hat nur eine periphere Operation durchgemacht, und dennoch entschloß man sich, sofort intrakraniell an die Ausrottung des Ganglions zu machen. $\mathrm{Zu}$ diesem Handeln führten uns folgende Überlegungen. Technisch ist der intrakranielle Eingriff sicher nicht schwieriger als die Operation an der Basis, dann aber mußten wir bei dem Symptomenbilde von vornherein überzeugt sein, daß wir es mit zentralen Störungen zu tun hatten. Wir haben wieder eine Mitbeteiligung sympathischer Fasern beobachten können, dazu kam die ganz erheblich auffällige einseitige Störung der Geschmackssensationen, auf den vorderen zwei Dritteln der linken Zungenseite, die nur durch eine Affektion proximal vom Ganglion sphenopalatinum im Ramus secundus, im Ganglion Gasseri selbst, oder 
im gemeinsamen Trigeminusstamm gesucht werden konnte. Es zeigte sich aber auch der Ramus I, wenn auch nicht sensibel, so doch sensorisch von der Affektion betroffen, indem die Geschmacksqualitäten eine Änderung im Sinne einer Verminderung erfahren hatten. In diesem Falle wäre eine weitere periphere Operation von vornherein aussichtslos gewesen.

So entschloß man sich, in Anbetracht des noch relativ guten Zustandes des Patienten zu dem eingreifenden radikalen intrakraniellen Verfahren. Hierbei stellten sich solche Schwierigkeiten entgegen, daß kein Erfolg beschieden war.

Schon die Blutung aus der Diploe war eine eminente, kaum zu stillende, je mehr man jedoch an der Dura arbeiten mußte, desto beträchtlicher wurde die Blutung, so daß man nur mit Mühe und großer Geduld ihrer Herr werden konnte. Wir sind geneigt, diese Größe der Blutung auf die Veränderungen der Gefäße zurückzuführen. Durch die Arteriosklerose waren die GefäBwände so stabil gemacht, daß weder ein Einrollen der Intima, noch eine Thrombusbildung möglich war, und die Gefäßwände der kleinen Duralgefäße äußerst brüchig waren.

Diese Gefäßveränderungen sind aber nicht nur an der Blutung, sondern auch an dem letalen Ende schuldig, die typische im Thalamus opticus und im occipitalen Teile der Capsula interna vorhandene innere Blutung mag wohl zum größten Teil am Tode schuldig gewesen sein. Ob diese indirekt auf den Spateldruck zurückzufuhren ist, oder ob durch das Hantieren am Gehirne durch die Unterbindung der Carotis interna Atheromstücke losgelöst wurden und so zur Apoplexie führten, ist eine Frage, die unbeantwortet bleiben muB.

Der durch den Spateldruck am Temporallappen erzeugte Erweichungsherd dürte kaum allein die Todesursache abgegeben haben. Wir sind gewohnt, bei komplizierten und unkomplizierten Schädelfrakturen viel schwerere Läsionen mit Wegfall ganzer großer Gehirnpartien zu sehen, ohne daß so schwere Symptome oder gar Exitus auftrat, wie im vorliegenden Falle.

Die zeitliche Länge und die Größe des Eingriffs, der starke Blutverlust, vor allem aber die Arteriosklerose haben in dieser Beobachtung, begünstigt durch eine Pleuritis und Bronchopneumonie, den Tod verursacht. 
Kasuistischer Beitrag zur operat. Behandlung der Trigeminusneuralgie usw. 97

Wenn wir eine Lehre aus dem Falle ziehen möchten, so ist es die, da $B$ Arteriosklerose die Ganglionexstirpation kontraindiziert. Sie vermehrt die Blutung, das Gehirn ist dem Spateldruck gegenüber widerstandsloser; vor allem aber ist die Gefahr von apoplektischen Insulten eine sehr große. Dies geht aus der von

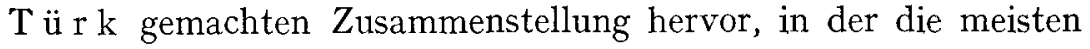
Todesfälle nach der Ganglionexstirpation von apoplektischen Insulten begleitet waren.

\section{Literaturverzeichnis.}

Die Literatur findet sich vollständig zusammengestellt in:

I. Le xer. Langenbecks Archiv, Bd. 65.

2. Hulles. Wiener klin. Wochenschr. I909, Nr. 27.

3. Räs umowsky. Langenbecks Archiv, Bd. 89 .

4. Trendele n burg, Verletzungen und chirurg. Krankheiten des Gesichtes. Deutsche Chir., Lief. 33, I. Teil. 